\title{
Autosomal dominant adult-onset proximal spinal muscular atrophy
}

INSERM

\section{Source}

INSERM. (1999). Orphanet: an online rare disease and orphan drug data base. Autosomal dominant adult-onset proximal spinal muscular atrophy. ORPHA:209335

A rare, genetic, motor neuron disease characterized by adulthood-onset of slowly progressive, proximal muscular weakness with fasciculations, amyotrophy, cramps, and absent/hypoactive reflexes, without bulbar or pyramidal involvement. 